\title{
Study on the Convective Term Discretized by Strong Conservation and Weak Conservation Schemes for Incompressible Fluid Flow and Heat Transfer
}

\author{
Peng Wang, Bo Yu, Jianyu Xie, Yu Zhao, Jingfa Li, and Qianqian Shao \\ National Engineering Laboratory for Pipeline Safety, Beijing Key Laboratory of Urban Oil and Gas Distribution Technology, \\ China University of Petroleum, Beijing 102249, China
}

Correspondence should be addressed to Bo Yu; yubobox@vip.163.com

Received 25 March 2013; Accepted 11 August 2013

Academic Editor: Shuyu Sun

Copyright (C) 2013 Peng Wang et al. This is an open access article distributed under the Creative Commons Attribution License, which permits unrestricted use, distribution, and reproduction in any medium, provided the original work is properly cited.

\begin{abstract}
When the conservative governing equation of incompressible fluid flow and heat transfer is discretized by the finite volume method, there are various schemes to deal with the convective term. In this paper, studies on the convective term discretized by two different schemes, named strong and weak conservation schemes, respectively, are presented in detail. With weak conservation scheme, the convective flux at interface is obtained by respective interpolation and subsequent product of primitive variables. With strong conservation scheme, the convective flux is treated as single physical variable for interpolation. The numerical results of two convection heat transfer cases indicate that under the same computation conditions, discretizing the convective term by strong conservation scheme would not only obtain a more accurate solution, but also guarantee the stability of computation and the clear physical meaning of the solution. Especially in the computation regions with sharp gradients, the advantages of strong conservation scheme become more apparent.
\end{abstract}

\section{Introduction}

In the 1980s, the conservation form and nonconservation form of the governing equation formally existed in the literature $[1,2]$. Since then, the governing equations are clearly classified into these two different forms, and for the control volume, the different characteristics of these two forms of equations gradually drew the attention of researchers. Based on previous studies, the conservation form of the governing equation is superior to the non-conservation form in many aspects, for example: (1) in the control volume of limited size, only the conservation form could ensure that the conservation principles of variables are satisfied [3-5]; (2) in the calculation of flow problem involving shock wave, the obtained flow field is usually smooth and stable, employing the conservation form of the governing equation; while using the non-conservation equation might lead to unsatisfactory spatial oscillations in the upstream and downstream regions of the shock wave [6-8]; (3) when the conservation equation is used in a body-fitted coordinate system, the conservativeness of the governing equation can still be satisfied $[9,10]$. More related research and applications are presented in the literature [11-18].

As a result, the conservation form of governing equation has been widely applied in the fields of computational fluid dynamics (CFD) and numerical heat transfer (NHT). In the compressible flow problems of aerodynamics, especially the flow containing shock wave, the conservation governing equation is usually expressed by first-order derivative equation system of the flux column vector, which is called strong conservation equation [1]. The convective flux in the strong conservation equation is regarded as a conservative variable. However, in the conservation equation employed in the incompressible fluid flow and heat transfer, the convective flux is the multiplication of primitive variables. In the aerodynamics field, when the governing equation is discretized by the finite volume method, strong conservation scheme is usually employed to deal with the convective flux 
TABle 1: Discretized expressions of the convective flux at the interface $(\rho A u \phi)_{e}$.

\begin{tabular}{|c|c|c|c|}
\hline Discrete format & Weak conservation scheme & Strong conservation scheme & Condition \\
\hline \multirow[b]{2}{*}{ FUD } & $\rho_{e} A_{e} u_{e} \phi_{P}$ & $(\rho A u \phi)_{P}$ & $u_{e} \geq 0$ \\
\hline & $\rho_{e} A_{e} u_{e} \phi_{E}$ & $(\rho A u \phi)_{E}$ & $u_{e}<0$ \\
\hline \multirow[b]{2}{*}{ SUD } & $\rho_{e} A_{e} u_{e} \frac{3 \phi_{P}-\phi_{W}}{2}$ & $\frac{3(\rho A u \phi)_{P}-(\rho A u \phi)_{W}}{2}$ & $u_{e} \geq 0$ \\
\hline & $\rho_{e} A_{e} u_{e} \frac{3 \phi_{E}-\phi_{E E}}{2}$ & $\frac{3(\rho A u \phi)_{E}-(\rho A u \phi)_{E E}}{2}$ & \multirow[t]{2}{*}{$u_{e}<0$} \\
\hline $\mathrm{CD}$ & $\rho_{e} A_{e} u_{e} \frac{\phi_{P}+\phi_{E}}{2}$ & $\frac{(\rho A u \phi)_{P}+(\rho A u \phi)_{E}}{2}$ & \\
\hline \multirow[b]{2}{*}{ QUICK } & $\rho_{e} A_{e} u_{e} \frac{3 \phi_{E}+6 \phi_{P}-\phi_{W}}{8}$ & $\frac{(3 \rho A u \phi)_{E}+6(\rho A u \phi)_{P}-(\rho A u \phi)_{W}}{8}$ & $u_{e} \geq 0$ \\
\hline & $\rho_{e} A_{e} u_{e} \frac{3 \phi_{P}+6 \phi_{E}-\phi_{E E}}{8}$ & $\frac{(3 \rho A u \phi)_{P}+6(\rho A u \phi)_{E}-(\rho A u \phi)_{E E}}{8}$ & $u_{e}<0$ \\
\hline
\end{tabular}

at interface as a whole, in order to guarantee the physical meaning of the numerical solution. While in the incompressible fluid flow and heat transfer field, the convective flux at interface is obtained by respective interpolation and subsequent product of relevant variables, which is called weak conservation scheme, correspondingly. The employment of weak conservation scheme is quite common in solving the incompressible fluid flow and heat transfer problems, and nevertheless, the applications of strong conservation scheme are rarely presented in the literature.

In order to yield a more reasonable discrete scheme of the convective term in the numerical computation of incompressible fluid flow and heat transfer, two common categories of the convection heat transfer problem are studied in this paper. Based on the discretization equation obtained by the finite volume method, the convective flux is discretized by strong and weak conservation schemes, respectively, and through the numerical results of cases, the advantages and disadvantages of these two different schemes are compared comprehensively and systematically in terms of accuracy, stability, and so on.

\section{Numerical Method}

It is noteworthy that the two discrete schemes of the convective term are derived from the finite volume method of the conservation governing equation, and thus, in the following text, the governing equations are all conservative and the discrete methods are all finite volume methods.

For the convenience of comparison and analysis without losing generality, the present paper takes the onedimensional convection heat transfer problem as an example, and the governing equation of this problem can be written as follows:

$$
\frac{\partial(\rho \phi)}{\partial t}+\frac{1}{A} \frac{\partial(\rho A u \phi)}{\partial x}=\frac{1}{A} \frac{\partial}{\partial x}\left(\Gamma A \frac{\partial \phi}{\partial x}\right),
$$

where the second term on the left-hand side $(1 / A)(\partial(\rho A u \phi) /$ $\partial x)$ is the convective term and its discretization is one critical

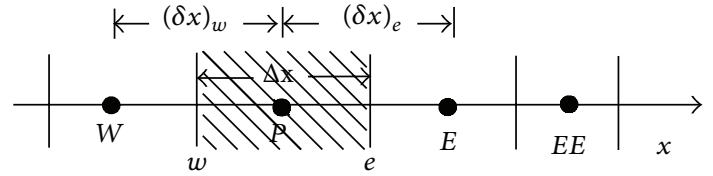

FIGURE 1: Sketch map of discrete grid.

key of the numerical computation. As mentioned above, in the numerical calculation of aerodynamics, strong conservation scheme is broadly employed to treat the convective flux $\rho A u \phi$ as a conservative variable. On the other hand, in the calculation of incompressible fluid flow and heat transfer, using weak conservation scheme, the primitive variables $\rho, A, u$, and $\phi$ are often discretized first and multiplied together to get the discretized convective term. For the sketch map of the discrete grid, the common expressions of the convective flux $(\rho A u \phi)_{e}$ at the interface $e$ with two different discrete schemes are shown in Table 1.

Through Table 1, with strong conservation scheme, the variable values of the convective flux at the interface are stored in the grid nodes rather than interpolate primitive variables at the interface. In the actual computation, this operation not only reduces the workload of interpolation to diminish the interpolation error, but also reduces the interface information storage to save memory.

In addition, the strong conservation scheme of the onedimensional problem is also suitable for multidimensional issues. It is well known that in the field of CFD and NHT, the general governing equation can be expressed as follows:

$$
\frac{\partial(\rho \phi)}{\partial t}+\operatorname{div}(\rho \mathbf{U} \phi)=\operatorname{div}\left(\Gamma_{\phi} \operatorname{grad} \phi\right)+S_{\phi}
$$

When the convective term $\operatorname{div}(\rho \mathbf{U} \phi)$ is discretized by strong and weak conservation schemes, respectively, the discrete expressions in the $x$ direction of the Cartesian coordinate system are the same with that in Table 1 . And it needs to be noted that $A_{e}=1$. 


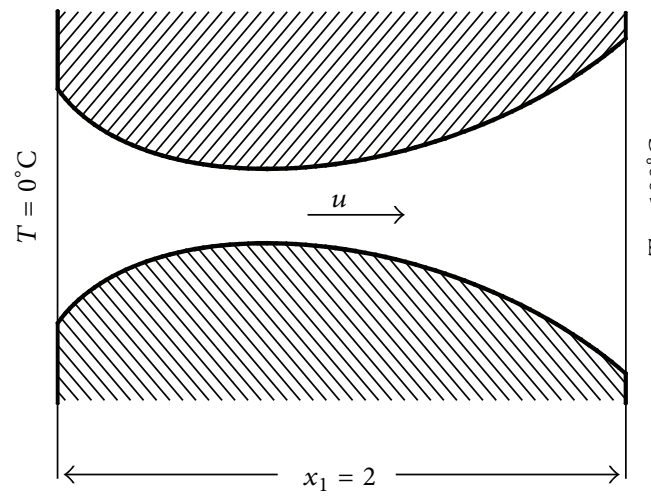

(a) One-dimensional problem

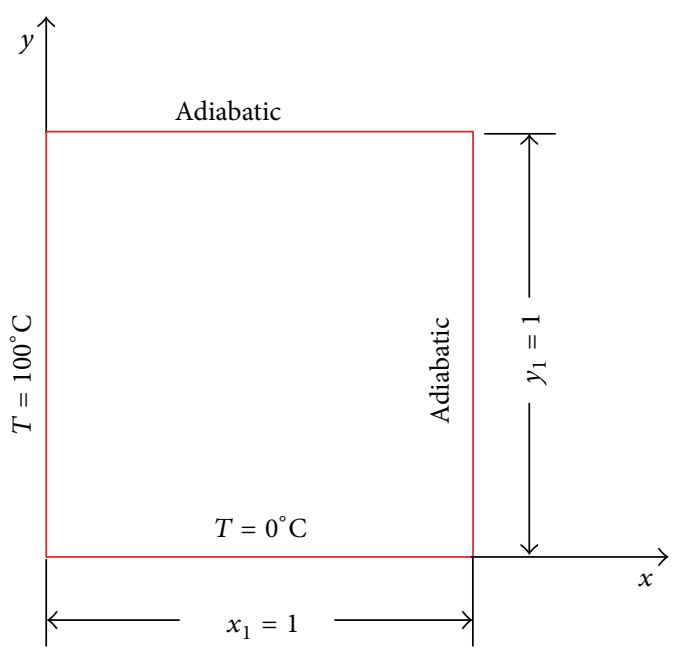

(b) Two-dimensional problem

FIGURE 2: Boundary conditions of two categories of the problem.

\section{Results and Analyses}

In order to compare the differences between the two conservation schemes in terms of computation accuracy and stability, the present paper first compares the numerical results of two categories of convection-diffusion cases: (1) the one-dimensional convection heat transfer problem; (2) the two-dimensional convection heat transfer problem with constant cross-section. Subsequently, Taylor series are used to analyze the truncation errors of the discretization equation with the convective term discretized by strong and weak conservation schemes, respectively. Finally, the deviations of the numerical solutions are presented to compare the computation accuracy of these two schemes.

3.1. Case Descriptions. The physical model of the onedimensional convection heat transfer problem (called "onedimensional problem" for short, hereinafter) is shown in Figure 2(a). The cases of this category, CASE 1 and CASE 2 (the governing equations are shown as (1)), correspond to two situations which have large gradient and relatively small gradient of primitive variables, respectively. Similarly, the physical model of the two-dimensional convection heat transfer problem with constant cross-section (called "twodimensional problem" for short, hereinafter) is shown in Figure 2(b). The cases of this category, CASE 3 and CASE 4 (the governing equations are shown as (2)), correspond to two situations which have large gradient and relatively small gradient of primitive variables, respectively.

The detailed boundary conditions of these two categories of the problem are presented in Figure 2, in which the left and right boundaries of the one-dimensional problem are of the first boundary condition, and the left and bottom boundaries of the two-dimensional problem are of the first boundary condition; meanwhile, the upper and right boundaries are of the second boundary condition. Table 2 shows the expressions of flow area and velocity distribution of the
TABLE 2: Flow area and velocity expressions of the one-dimensional problem.

\begin{tabular}{ccc}
\hline & Flow area & Velocity $u$ \\
\hline CASE 1 & $A=-\sin (\pi x / 2)+1.5$ & $u=0.2 /(-\sin (\pi x / 2)+1.5)$ \\
CASE 2 & $A=4(-\sin (\pi x / 2)+1.01)$ & $u=0.05 /(-\sin (\pi x / 2)+1.01)$ \\
\hline
\end{tabular}

TABLE 3: Velocity expressions of the two-dimensional problem.

\begin{tabular}{ccc}
\hline & Velocity $u$ & Velocity $v$ \\
\hline CASE 3 & $u=0.1 \sin (2 \pi(x+y))$ & $v=-0.1 \sin (2 \pi(x+y))$ \\
CASE 4 & $u=\sin (2 \pi(x+y))$ & $v=-\sin (2 \pi(x+y))$ \\
\hline
\end{tabular}

two one-dimensional cases. And the velocity profiles in the $x$ direction of the two one-dimensional cases are given in Figures 3(a) and 3(b). In Table 3, there are the expressions of velocity distribution of the two two-dimensional cases. For direct display of the velocity distribution, Figures 3(c) and 3 (d) depict the $u$ and $v$ velocity distributions in the position of $y=0.5$ along the $x$ direction. It is noteworthy that in the four cases, densities and thermal conductivities are all set to be $\rho=1000\left(\mathrm{~kg} / \mathrm{m}^{3}\right)$ and $\Gamma=100 \mathrm{~W} /\left(\mathrm{m} \cdot{ }^{\circ} \mathrm{C}\right)$, respectively.

Different kinds of uniform mesh are utilized for these four cases, and the grid number of CASE 3 and CASE 4 in the $x$ and $y$ directions is the same, that is, $N_{x}=N_{y}$. The convective term is discretized by weak conservation scheme and strong conservation scheme, respectively, and in the following text, these two schemes are referred to as Scheme 1 and Scheme 2. CD and QUICK formats are also employed to discretize the governing equation. The stable field of variable $\phi$ is obtained by solving the discretized equation with explicit time marching method. And the numerical results indicate that all four cases achieve the grid-independent solutions when the grid number satisfies $N_{x}=200$. 


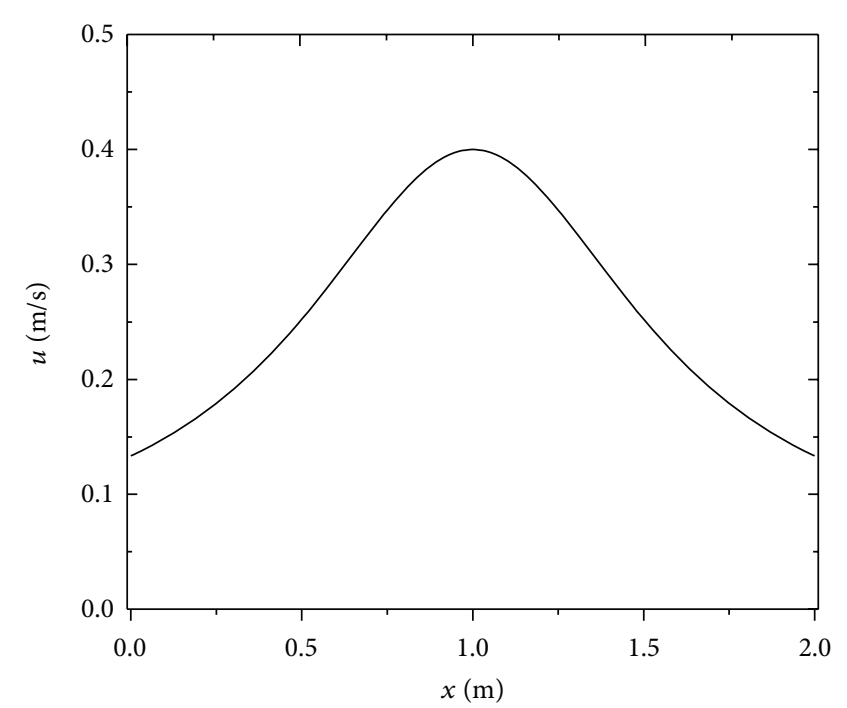

(a) CASE 1

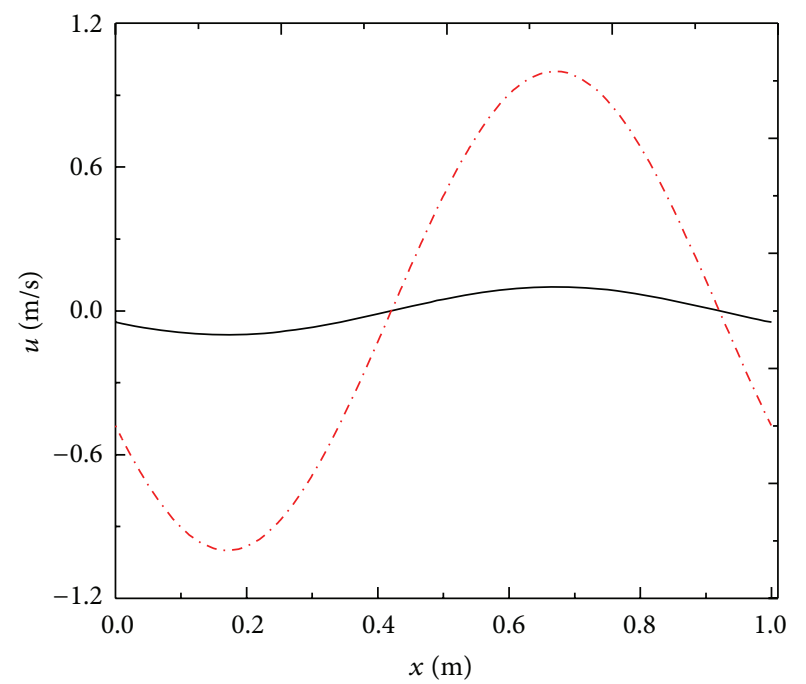

CASE 3

-... CASE 4

(c) Distribution of velocity $u$ in $y=0.5$

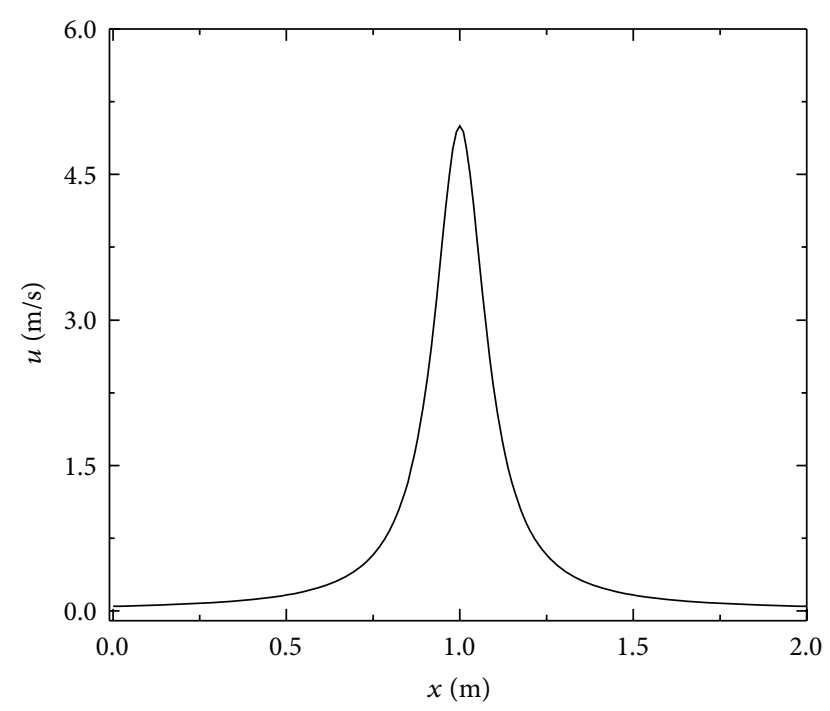

(b) CASE 2

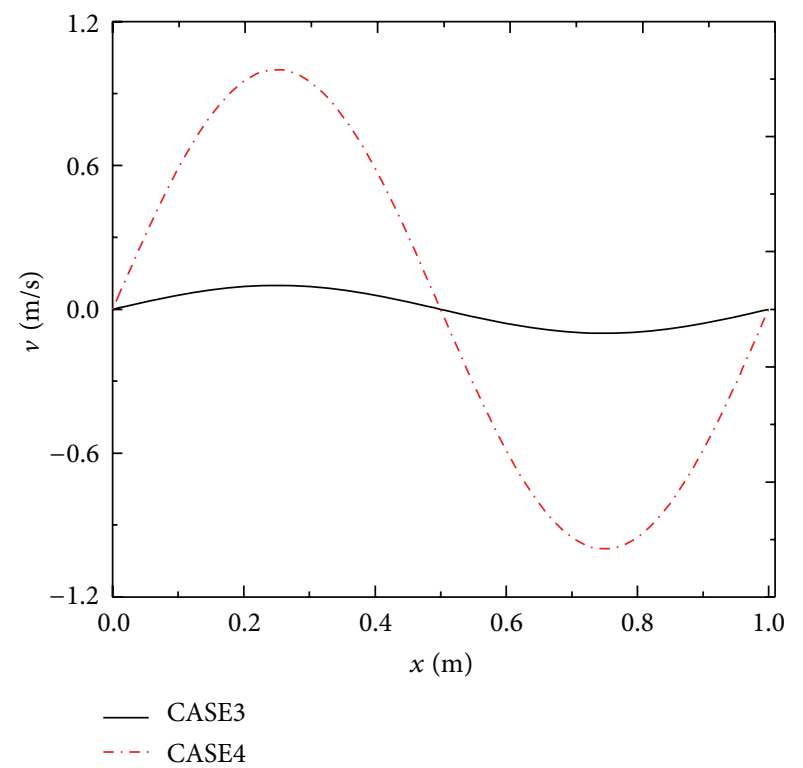

(d) Distribution of velocity $v$ in $y=0.5$

Figure 3: Velocity distribution along the $x$ direction.

3.2. Numerical Results. As shown in Figure 4, the present paper first compares the solution of the two schemes on the sparse grid $\left(N_{x}=7\right.$ for the one-dimensional problem and $N_{x}=30$ for the two-dimensional problem) with the gridindependent solution.

In Figure 4, Figures 4(a) to 4(d) show the temperature distribution along the $x$ coordinate in CASE 1 and CASE 2 with CD and QUICK formats, and Figures 4(e) to 4(h) present the contour map of temperature field in CASE 3 and CASE 4 with CD and QUICK formats. It is found that on the sparse grid when the gradient of variables is relatively small, the solutions of these two schemes agree with the grid-independent solution very well. However, when the gradient becomes relatively large, the solution with strong conservation scheme is obviously better than that with weak conservation scheme. It can be seen from Figures 4(b) and 4(d) that in CASE 2, when the convective term is discretized by weak conservation scheme, the solution would overshoot in the position of $x=1.0$, and with the CD format, the oscillations are even more severe. In contrast, the solution of clear physical meaning can be obtained with strong conservation scheme. As shown in Figures 4(f) and 4(h), in the top right corner of the temperature contour map, the solution with weak conservation scheme deviates greatly from the grid-independent solution.

Through the above analyses, under the same computation conditions, when the primitive variables change slightly, the difference between the solutions of the two schemes is very 


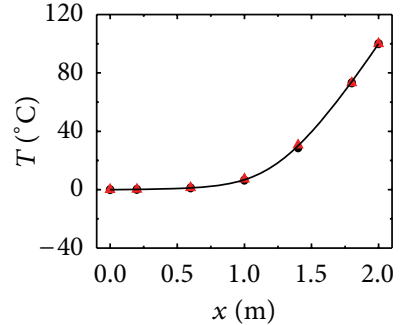

- Scheme 1

- Scheme 2

- Grid-independent solution

(a) CASE 1, CD format

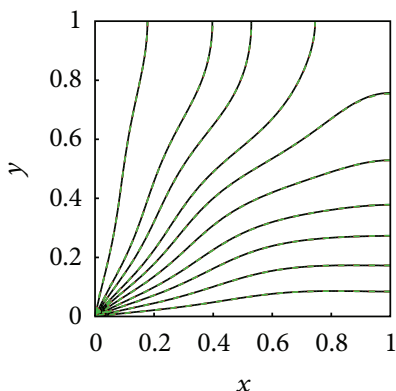

- - Scheme 1

-... Scheme 2

- Grid-independent solution

(e) CASE 3, CD format

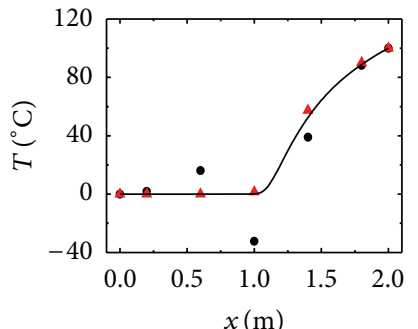

- Scheme 1

- Scheme 2

- Grid-independent solution

(b) CASE 2, CD format

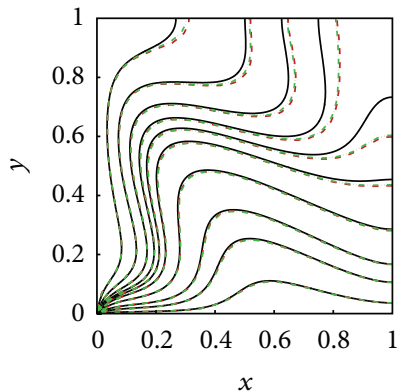

- - - Scheme 1

- - . Scheme 2

- Grid-independent

solution

(f) CASE 4, CD format

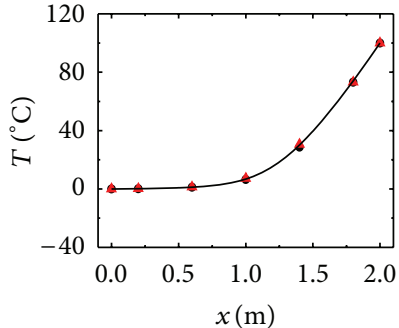

- Scheme 1

- Scheme 2

— Grid-independent solution

(c) CASE 1, QUICK format

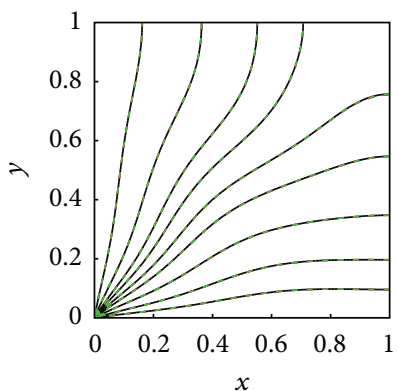

- - - Scheme 1

-... Scheme 2

- Grid-independent solution

(g) CASE 3, QUICK format

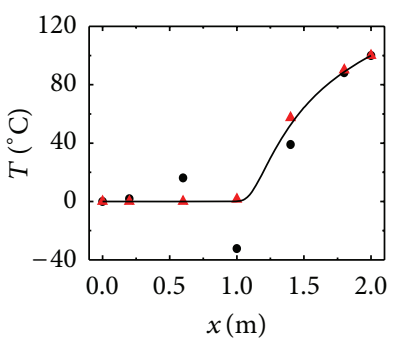

- Scheme 1

- Scheme 2

- Grid-independent solution

(d) CASE 2, QUICK format

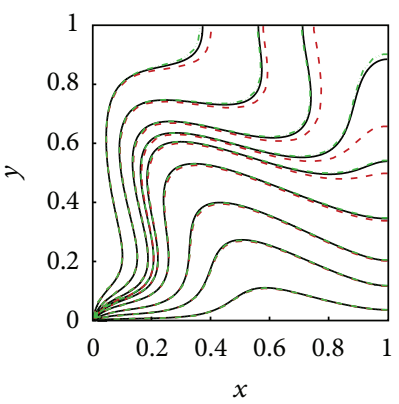

- - - Scheme 1

-... Scheme 2

— Grid-independent solution

(h) CASE 4, QUICK format

FIGURE 4: Comparison of different evaluations of the convective term.

small, but when there are sharp changes of variables, the superiority of high accuracy and stability with strong conservation scheme is apparently shown, and the corresponding solution is more likely to have clear physical meaning.

3.3. Accuracy Analysis. In order to further compare the accuracy difference of the numerical solutions with strong and weak conservation schemes, the present paper introduces Taylor series to analyze the truncation error of the same computation form obtained by discretizing the convective term with two different schemes. Taking the $\mathrm{CD}$ format of CASE 1 and CASE 2 as an example to explain the following.

Scheme 1:

$$
\begin{aligned}
\frac{\rho_{P}+\rho_{E}}{2} & \frac{A_{P}+A_{E}}{2} \frac{u_{P}+u_{E}}{2} \frac{T_{P}+T_{E}}{2} \\
= & \rho_{e} A_{e} u_{e} T_{e}+\left.\rho_{e} A_{e} T_{e} \frac{1}{8} \frac{\partial^{2} u}{\partial x^{2}}\right|_{e} \Delta x^{2} \\
& +\left.\rho_{e} u_{e} T_{e} \frac{1}{8} \frac{\partial^{2} A}{\partial x^{2}}\right|_{e} \Delta x^{2} \\
& +\left.\rho_{e} A_{e} u_{e} \frac{1}{8} \frac{\partial^{2} T}{\partial x^{2}}\right|_{e} \Delta x^{2}+o\left(\Delta x^{3}\right) .
\end{aligned}
$$

Scheme 2:

$$
\begin{aligned}
\frac{(\rho A u T)_{P}+(\rho A u T)_{E}}{2}= & (\rho A u T)_{e} \\
& +\left.\frac{1}{8} \frac{\partial^{2}(\rho A u T)}{\partial x^{2}}\right|_{e} \Delta x^{2}+o\left(\Delta x^{3}\right) .
\end{aligned}
$$

The truncation errors of $\mathrm{CD}$ format obtained by the two schemes can be expressed as:

$$
\begin{aligned}
\varepsilon^{1}= & \left.\rho_{e} A_{e} T_{e} \frac{1}{8} \frac{\partial^{2} u}{\partial x^{2}}\right|_{e} \Delta x^{2}+\left.\rho_{e} u_{e} T_{e} \frac{1}{8} \frac{\partial^{2} A}{\partial x^{2}}\right|_{e} \Delta x^{2} \\
& +\left.\rho_{e} A_{e} u_{e} \frac{1}{8} \frac{\partial^{2} T}{\partial x^{2}}\right|_{e} \Delta x^{2}+o\left(\Delta x^{3}\right), \\
\varepsilon^{2}= & \left.\frac{1}{8} \frac{\partial^{2}(\rho A u T)}{\partial x^{2}}\right|_{e} \Delta x^{2}+o\left(\Delta x^{3}\right) .
\end{aligned}
$$

Since the incompressible fluid flow satisfies the continuity equation, (6) can be further written as follows:

$$
\varepsilon^{2}=\left.(\rho A u)_{e} \frac{1}{8} \frac{\partial^{2} T}{\partial x^{2}}\right|_{e} \Delta x^{2}+o\left(\Delta x^{3}\right) .
$$




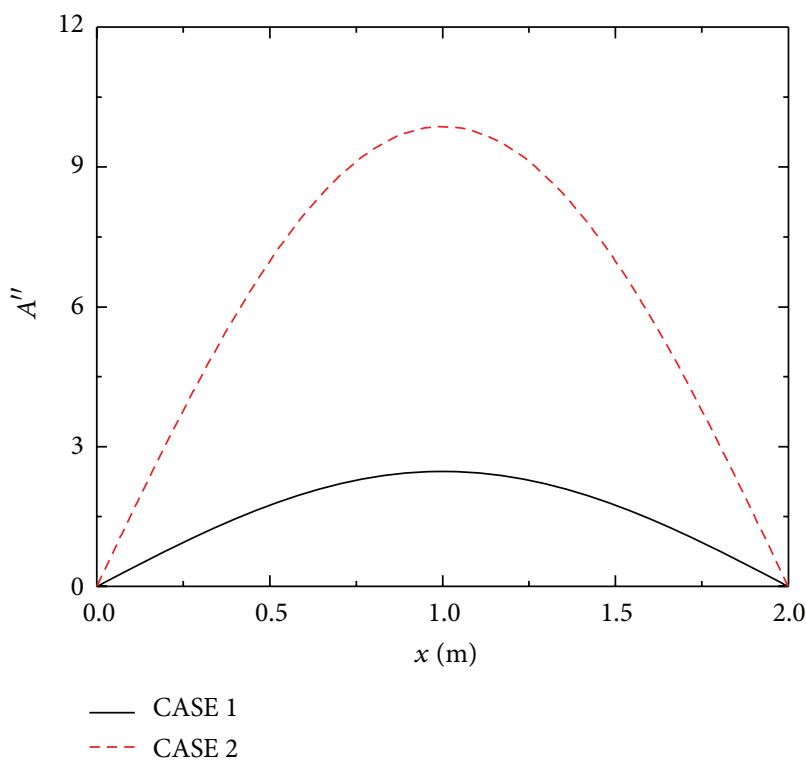

(a) Value of $\partial^{2} A / \partial x^{2}$

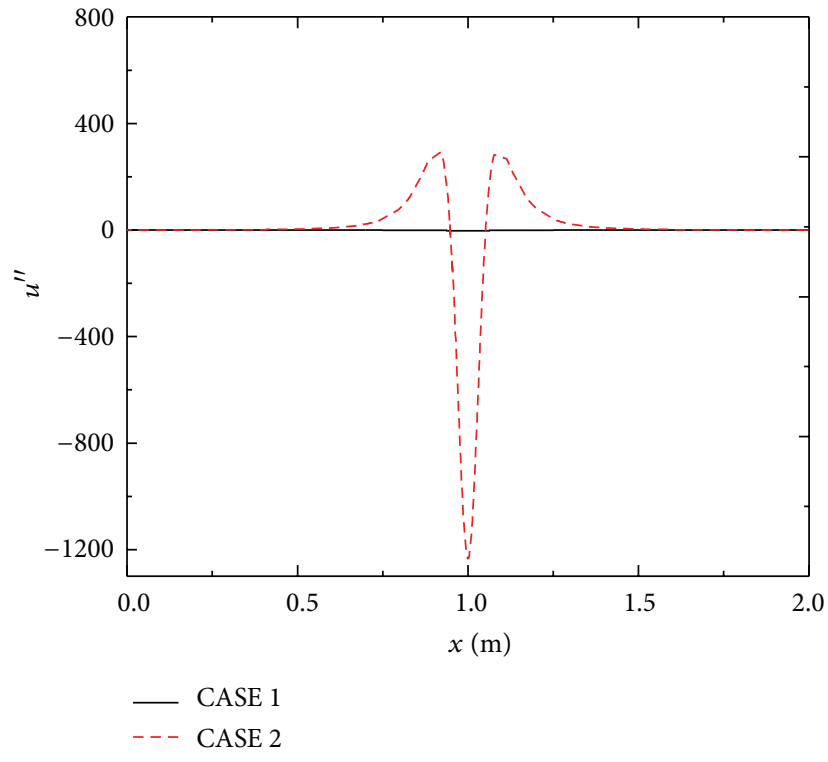

(b) Value of $\partial^{2} u / \partial x^{2}$

FIGURE 5: Values of $\partial^{2} A / \partial x^{2}$ and $\partial^{2} u / \partial x^{2}$ in CASE 1 and CASE 2.

Through comparing (5) and (6), the truncation error of weak conservation scheme contains $\partial^{2} A / \partial x^{2}$ and $\partial^{2} u / \partial x^{2}$, while these two terms, respectively, reflect the changing degree of flow area and velocity. In order to further analyze the influence of $\partial^{2} A / \partial x^{2}$ and $\partial^{2} u / \partial x^{2}$ on the errors $\varepsilon^{1}$ and $\varepsilon^{2}$, the values of $\partial^{2} A / \partial x^{2}$ and $\partial^{2} u / \partial x^{2}$ in one-dimensional cases are shown in Figure 5.

From Figure 5, the values of $\partial^{2} A / \partial x^{2}$ and $\partial^{2} u / \partial x^{2}$ in CASE 2 fluctuate more acutely than those in CASE 1 , especially in the position of $x=1.0$. The absolute value of $\partial^{2} u / \partial x^{2}$ in CASE 2 reaches as great as 1200 , which is far larger than that in CASE 1. Correspondingly, comparing Figures 4(b) and 4(d), we can see that the abrupt change of velocity in CASE 2 with Scheme 1 appears exactly in the position of $x=1.0$, and in the same position, the computation results of CASE 2 overshoot to make the solution not conform to the physical meaning. Hence, it is reasonable to conclude that in some cases, the values of $\partial^{2} A / \partial x^{2}$ and $\partial^{2} u / \partial x^{2}$ with weak conservation scheme have a serious effect on the numerical errors; however, there is no such problem with strong conservation scheme.

Based on the above analysis, the derivative of primitive variable is very small when the changing gradient is relatively small. When the values of the first two terms in (5) can be neglected compared with that of the third term, the truncation errors of the two different schemes are almost the same, and the two numerical solutions have no obvious difference. However, when the changing gradient is very large, the derivative of primitive variable becomes large, correspondingly. If the values of the first two terms in (5) are equal to or even greater than the value of the third term, it is obvious that $\varepsilon^{1}>\varepsilon^{2}$ and that there is a big difference between the numerical solutions of the two schemes.
Last but not least, in order to compare the calculation accuracy of the two different schemes quantitatively, the present paper defines the average absolute error and maximum absolute error as follows:

$$
\begin{aligned}
& \text { average absolute error: } \varepsilon_{a}=\frac{1}{N_{\text {Grid }}} \sum_{n=1}^{N_{\text {Grid }}}\left|T_{c}-T_{b}\right|, \\
& \text { maximum absolute error: } \varepsilon_{m}=\max \left(\left|T_{c}-T_{b}\right|\right) .
\end{aligned}
$$

Through (8), the average and maximum absolute errors of the four cases on different kinds of uniform mesh, in which the largest grid number is $N_{x}=200$ and the smallest one is $N_{x}=10$, are calculated. And the numerical solutions on the grid $N_{x}=1000$ and $N_{x}=500$ are, respectively, regarded as the benchmark solutions of the one-dimensional and two-dimensional problems. It is worth pointing out that the grid number of the benchmark solution taken in this paper is much larger than the required grid number of obtaining the grid-independent solution; thus, it is feasible to use these numerical solutions to evaluate the average and maximum absolute errors of the two different schemes with grid numbers ranging from $N_{x}=10$ to $N_{x}=200$. Additionally, the same conclusion can be obtained using CD or QUICK formats; thus, only the average and maximum absolute errors of the numerical solution employing QUICK format (as shown in Figures 6 and 7, resp.) are given to evaluate the computation accuracy. Furthermore, the ratios of the average absolute errors and those of the maximum absolute errors of the four cases employing QUICK format are presented in Figure 8.

From Figure 6, it is easily found that on the same grid, the average absolute errors of the numerical solutions with 


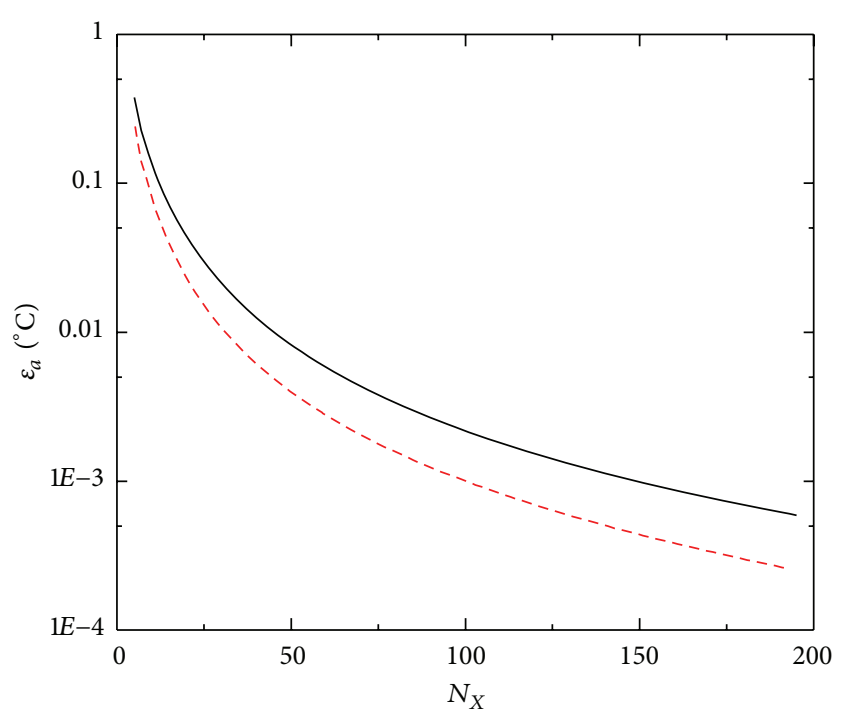

(a) CASE 1

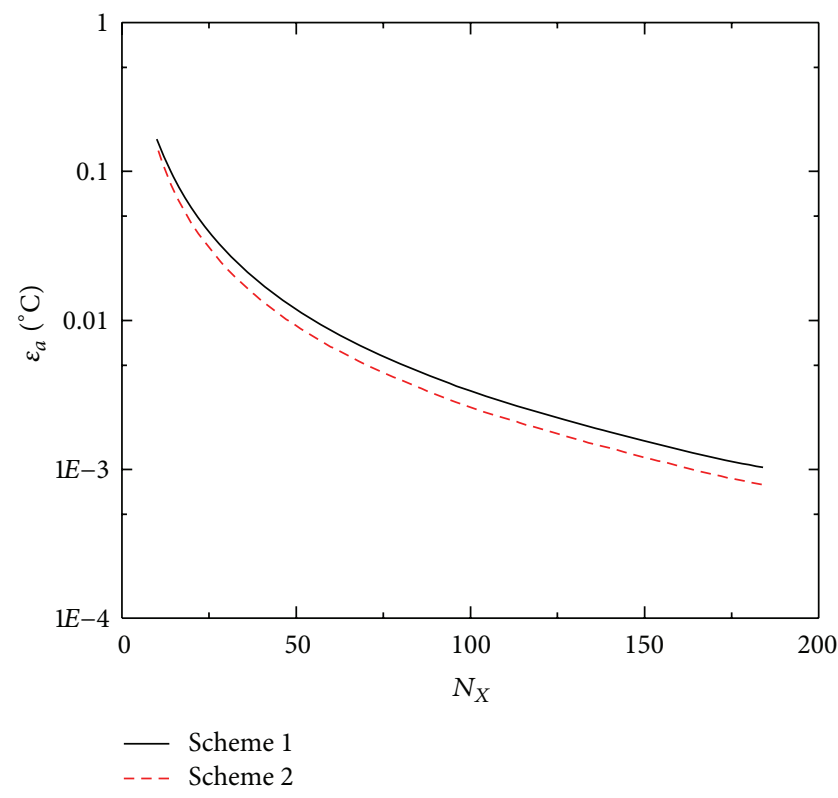

(c) CASE 3

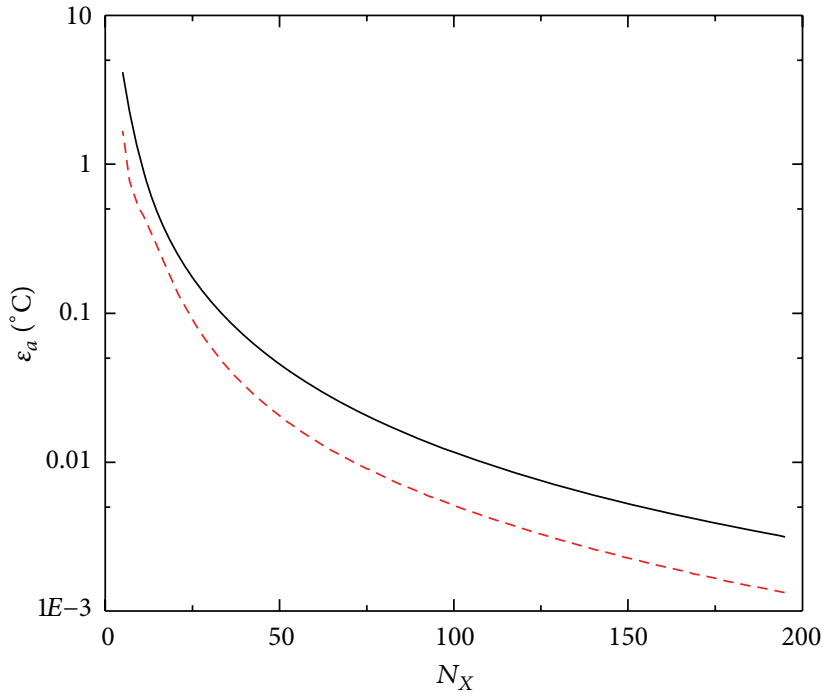

(b) CASE 2

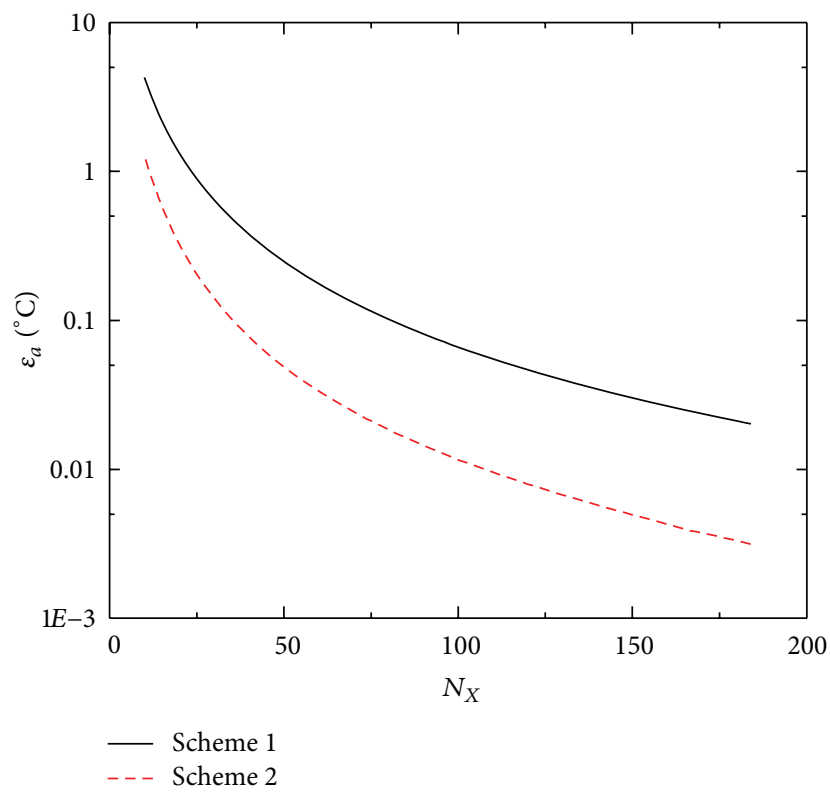

(d) CASE 4

FIGURE 6: Average absolute error.

strong conservation scheme are all less than those with weak conservation scheme in the four cases.

As shown in Figure 7, when it comes to dealing with the one-dimensional problem, Scheme 2 is superior to Scheme 1 , while in solving the two-dimensional problem, the performances of the two different schemes are basically the same. The reason why the results of CASE 3 and CASE 4 are not desirable lies in the consideration of the second boundary condition in CASE 3 and CASE 4. For the strong and weak conservation schemes, the maximum error usually occurs in the boundary adjacent nodes. Due to the fact that the effective treatment method of boundary condition has not been found so far, strong conservation scheme did not have an obvious advantage over weak conservation scheme in
CASE 3 and CASE 4. Comparing Figures 7(a), 7(b), 7(c), and $7(d)$, at the same time, it shows that when the gradient of variables is relatively large, in terms of average and maximum absolute errors, the solution with Scheme 2 enjoys higher accuracy than that with Scheme 1. That is because more interface interpolations are involved in Scheme 1, which might lead to error accumulation. Thus, under the same computation conditions, the solution with Scheme 1 is not as accurate as that with Scheme 2, and the larger the gradient of primitive variables is, the greater the errors due to interface interpolations are, along with the more obvious difference between the solutions of the two schemes.

Through Figure 8, the two kinds of ratio, average absolute error and maximum absolute error, with the two different 


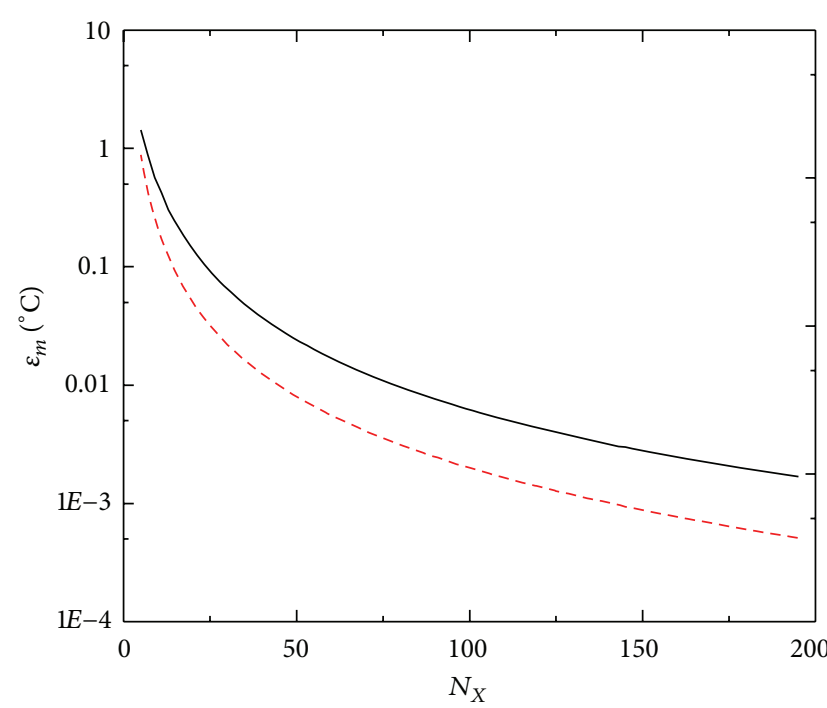

(a) CASE 1

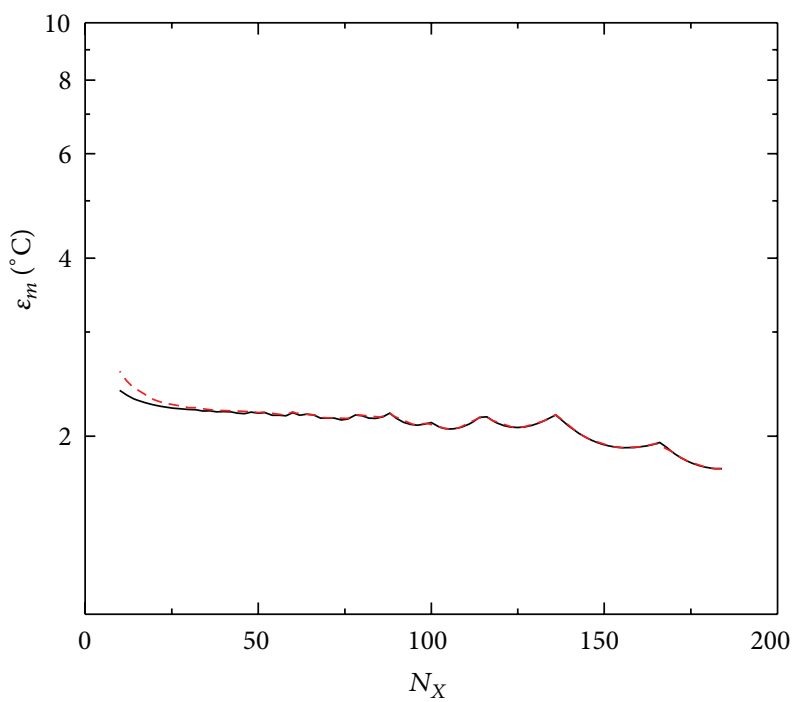

- Scheme 1

- - Scheme 2

(c) CASE 3

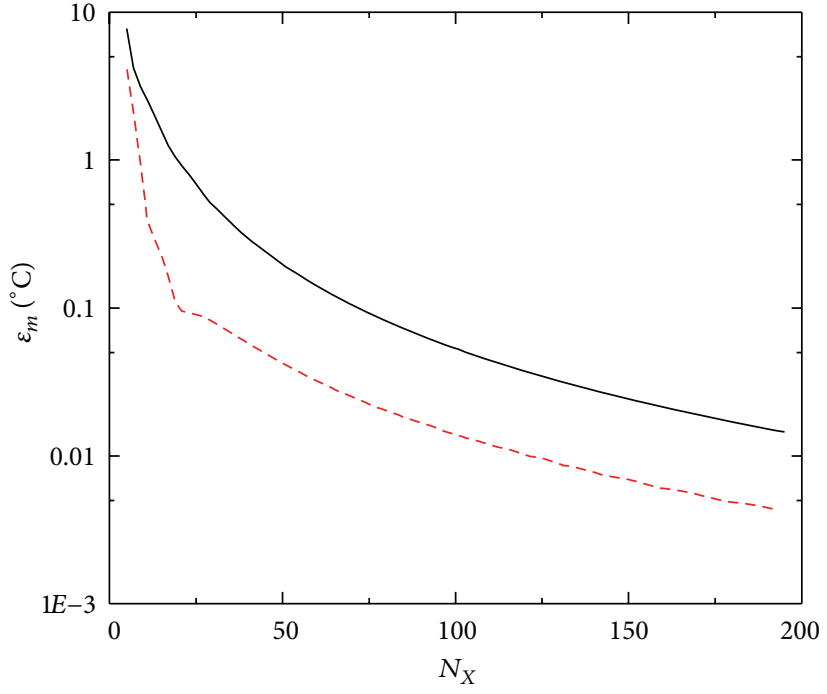

(b) CASE 2

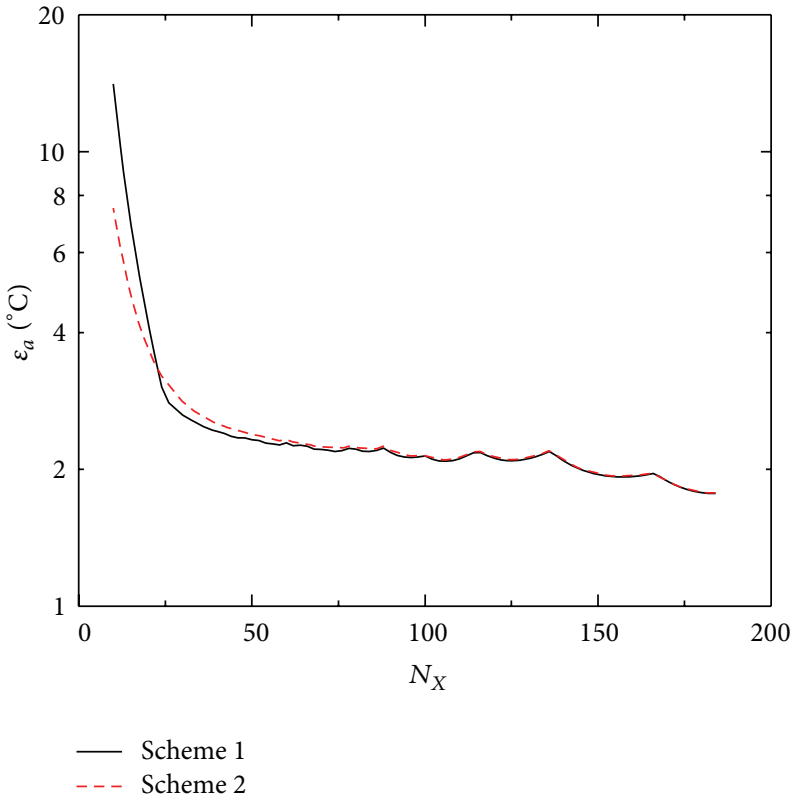

(d) CASE 4

FIGURE 7: Maximum absolute error.

schemes are basically larger than 1 . Notice specifically, from Figure 8 (a) that the ratios of average absolute errors with the two schemes in solving the one-dimensional problem are presented basically to be 2 , while in CASE 4 of the twodimensional problem, the ratios of average absolute errors can be as large as 4-7. Moreover, from Figure 8(b), in solving the one-dimensional problem, the maximum absolute error with Scheme 1 is almost three times greater than that with Scheme 2.

In short, under the same computation conditions, when the gradient of primitive variables is relatively large, the numerical solution with Scheme 2 is more accurate than that with Scheme 1.

\section{Conclusions}

In the incompressible fluid flow and heat transfer, when the conservation governing equation is discretized by the finite volume method, the convective flux at the interface can be obtained by strong conservation scheme and weak conservation scheme. The present paper studies the influences of these two different schemes on the numerical solution with various typical cases. The results of the numerical cases indicate the following:

(1) under the same computation conditions and on the sparse grid, when the gradient of primitive variables is relatively small, the numerical solutions with 


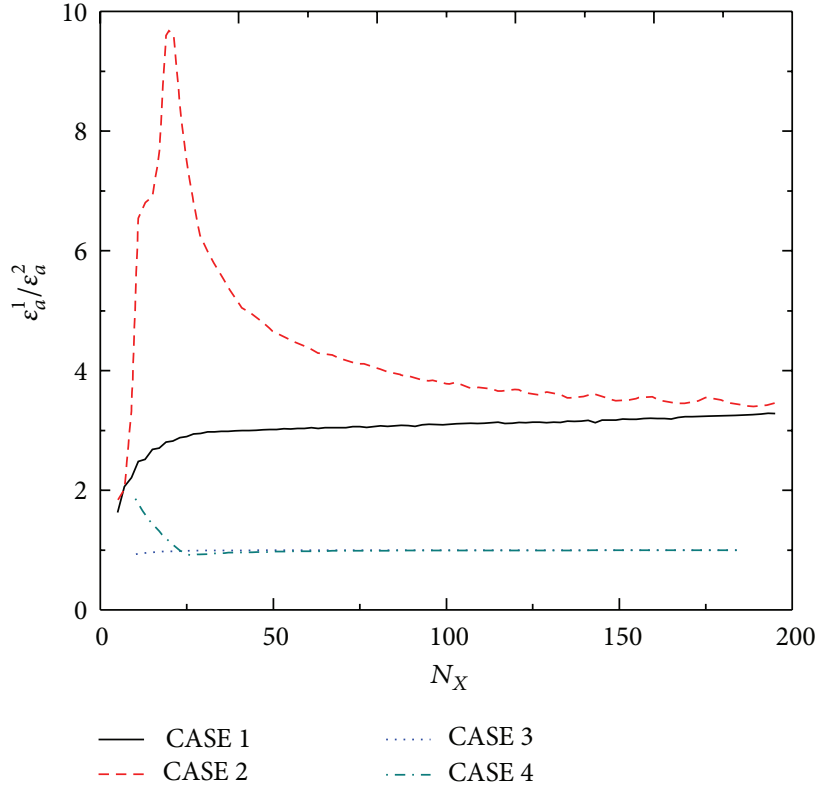

(a) Ratios of average absolute errors

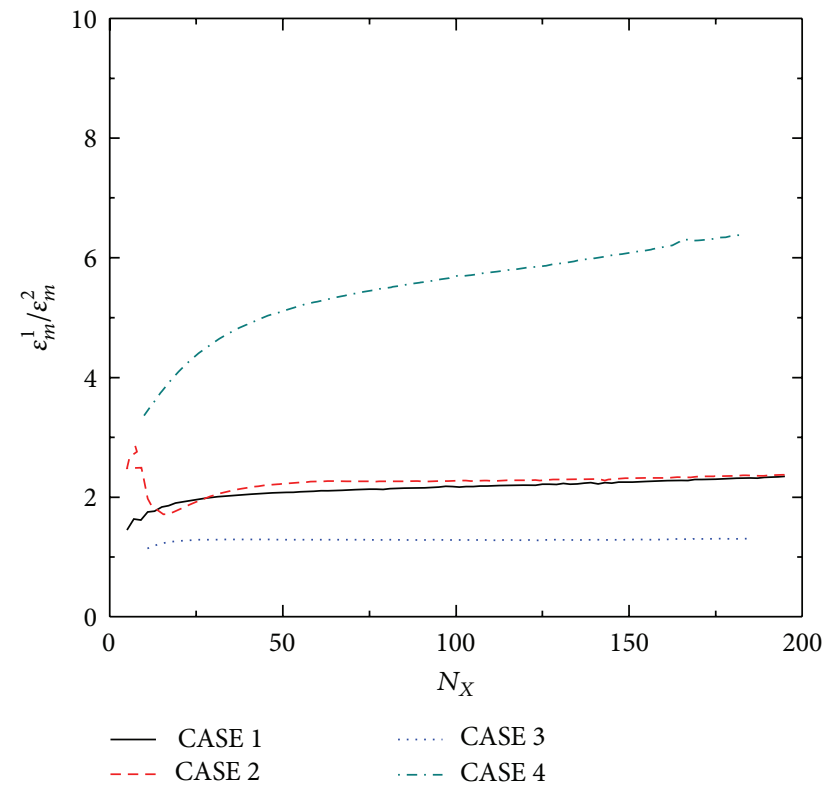

(b) Ratios of maximum absolute errors

FIGURE 8: Ratios of errors.

the two schemes are close to each other and all could match the grid-independent solution quite well. When the gradient becomes sharp, the solution with weak conservation scheme deviates greatly from the benchmark solution: even oscillation or solution without physical meaning occurs. On the other hand, the solution with strong conservation scheme still matches the grid-independent solution well;

(2) through calculating average absolute error and maximum absolute error, it is found that even when the gradient of primitive variables is small, strong conservation scheme still has advantage over weak conservation scheme.

In conclusion, treating the convective flux as single physical variable and discretizing the convective term by strong conservation scheme is a more effective way in the numerical computation of incompressible fluid flow and heat transfer.

\section{Nomenclatures}

$t: \quad$ Time, $\mathrm{s}$

$u, v: \quad$ Velocities, $\mathrm{m} / \mathrm{s}$

$u^{\prime \prime}$ : $\quad$ Second partial derivative of velocity

$u, u^{\prime \prime}=\partial^{2} u / \partial x^{2}$

$x, y: \quad$ Spatial coordinates, $\mathrm{m}$

U: $\quad$ Velocity vector

A: $\quad$ Flow area, $\mathrm{m}^{2}$

$A^{\prime \prime}$ : $\quad$ Second partial derivative of flow area $A, A^{\prime \prime}=\partial^{2} A / \partial x^{2}$

$N_{x}, N_{y}$ : Grid numbers in the $x$ and $y$ coordinates

$N_{\text {Grid }}$ : Total grid number

$S_{\phi}: \quad$ Heat source, $\mathrm{W} / \mathrm{m}^{3}$.

\section{Greek Symbols}

$\Delta x$ : Width of control volume in the $x$ direction, $\mathrm{m}$

$\delta x$ : Distance between adjacent nodes, $\mathrm{m}$

$\rho: \quad$ Density, $\mathrm{kg} / \mathrm{m}^{3}$

$\phi:$ General variable

$\varepsilon$ : Numerical error

$\Gamma_{\phi}:$ General diffusion coefficient, $\mathrm{W} /\left(\mathrm{m} \cdot{ }^{\circ} \mathrm{C}\right)$

$\Gamma$ : Thermal conductivity, $\mathrm{W} /\left(\mathrm{m} \cdot{ }^{\circ} \mathrm{C}\right)$.

\section{Superscripts}

1: Scheme 1, weak conservation scheme

2: Scheme 2, strong conservation scheme.

\section{Subscripts}

$\begin{array}{ll}a: & \text { Average error } \\ b: & \text { Benchmark solution } \\ c: & \text { Calculation solution } \\ l: & \text { Computational domain } \\ m: & \text { Maximum error } \\ e, w: & \text { Interfaces of the control volume } P \text { as } \\ P, W, E, E E: & \text { Grown in Figure 1 }\end{array}$

\section{Acknowledgment}

This study is supported by the National Science Foundation of China (nos. 51134006, 51276198, and 51176204). 


\section{References}

[1] J. D. Anderson, Computational Fluid Dynamics-the Basics with Applications, McGraw-Hill, New York, NY, USA, 1st edition, 1995.

[2] P. J. Roache, Computational Fluid Dynamics, Hermosa, 3rd edition, 1976.

[3] W. Q. Tao, Numerical Heat Transfer, Xi'an Jiaotong University Press, 2nd edition, 2002.

[4] W. Q. Tao, Advances in Computational Heat Transfer, Science Press, 2nd edition, 2000.

[5] H. K. Versteeg and W. Malalasekera, An Introduction to Computational Fluid Dynamics: The Finite Volume Method, Prentice Hall, 2nd edition, 2007.

[6] P. D. Lax, "Weak solutions of nonlinear hyperbolic equations and their numerical computation," Communications on Pure and Applied Mathematics, vol. 7, pp. 159-193, 1954.

[7] P. Lax and B. Wendroff, "Systems of conservation laws," Communications on Pure and Applied Mathematics, vol. 13, no. 2, pp. 217-237, 1960.

[8] A. Jameson, "Transonic potential flow calculations using conservation form," in Proceedings of the Computational Fluid Dynamics Conference, 75A34193, pp. 148-161, Hartford, Conn, USA, 1975.

[9] M. Vinokur, "Conservation equations of gasdynamics in curvilinear coordinate systems," Journal of Computational Physics, vol. 14, pp. 105-125, 1974.

[10] T. J. Bridges, "Conservation laws in curvilinear coordinates: a short proof of Vinokur's theorem using differential forms," Applied Mathematics and Computation, vol. 202, no. 2, pp. 882$885,2008$.

[11] R. J. DiPerna, "Decay of solutions of hyperbolic systems of conservation laws with a convex extension," Archive for Rational Mechanics and Analysis, vol. 64, no. 1, pp. 1-46, 1977.

[12] A. Bressan, "Hyperbolic systems of conservation laws," Revista Matemática Complutense, vol. 12, no. 1, pp. 135-200, 1999.

[13] R. K. S. Hankin, "The Euler equations for multiphase compressible flow in conservation form-Simulation of shock-bubble interactions," Journal of Computational Physics, vol. 172, no. 2, pp. 808-826, 2001.

[14] C. Lai, R. A. Baltzer, and R. W. Schaffranek, "Conservation-form equations of unsteady open-channel flow," Journal of Hydraulic Research, vol. 40, no. 5, pp. 567-578, 2002.

[15] L. M. Gao, K. T. Li, B. Liu, and Su J, "General integral conservation form of Navier-Stokes equations and numerical application," Chinese Journal of Computational Physics, vol. 25, no. 2, pp. 172-178, 2008.

[16] S. H. Sohrab, "Derivation of invariant forms of conservation equations from the invariant Boltzmann equation," in Proceedings of the 5th International Conference on Fluid Mechanics, pp. 27-35, Acapulco, Mexico, 2008.

[17] S.-F. Li, P. Zhang, and S. C. Wong, "Conservation form of Helbing's fluid dynamic traffic flow model," Applied Mathematics and Mechanics, vol. 32, no. 9, pp. 1109-1118, 2011.

[18] R. C. Martineau, R. A. Berry, A. Esteve et al., "Comparison of natural convection flows under VHTR type conditions modeled by both the conservation and incompressible forms of the Navier-Stokes equations," Nuclear Engineering and Design, vol. 240, no. 6, pp. 1371-1385, 2010. 


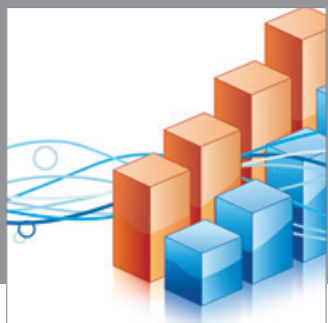

Advances in

Operations Research

mansans

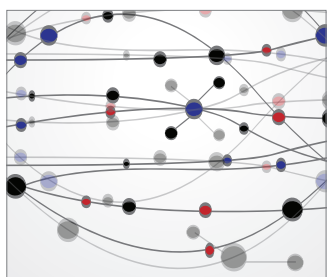

The Scientific World Journal
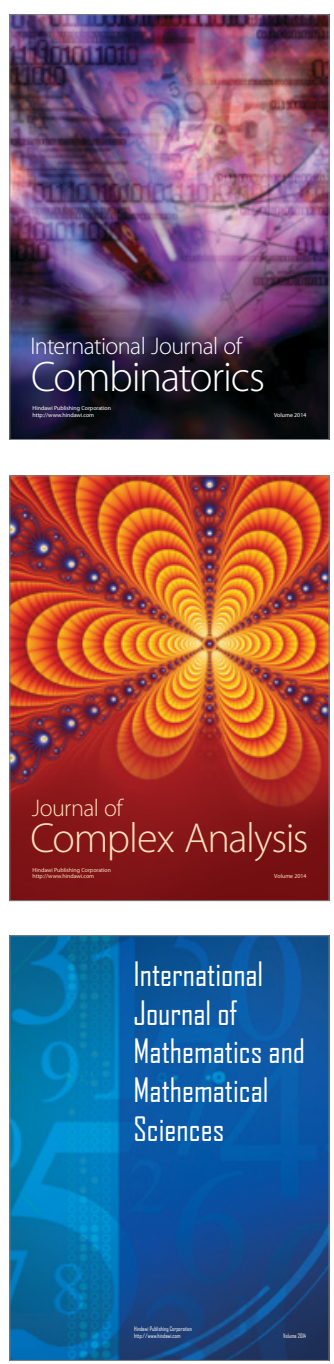
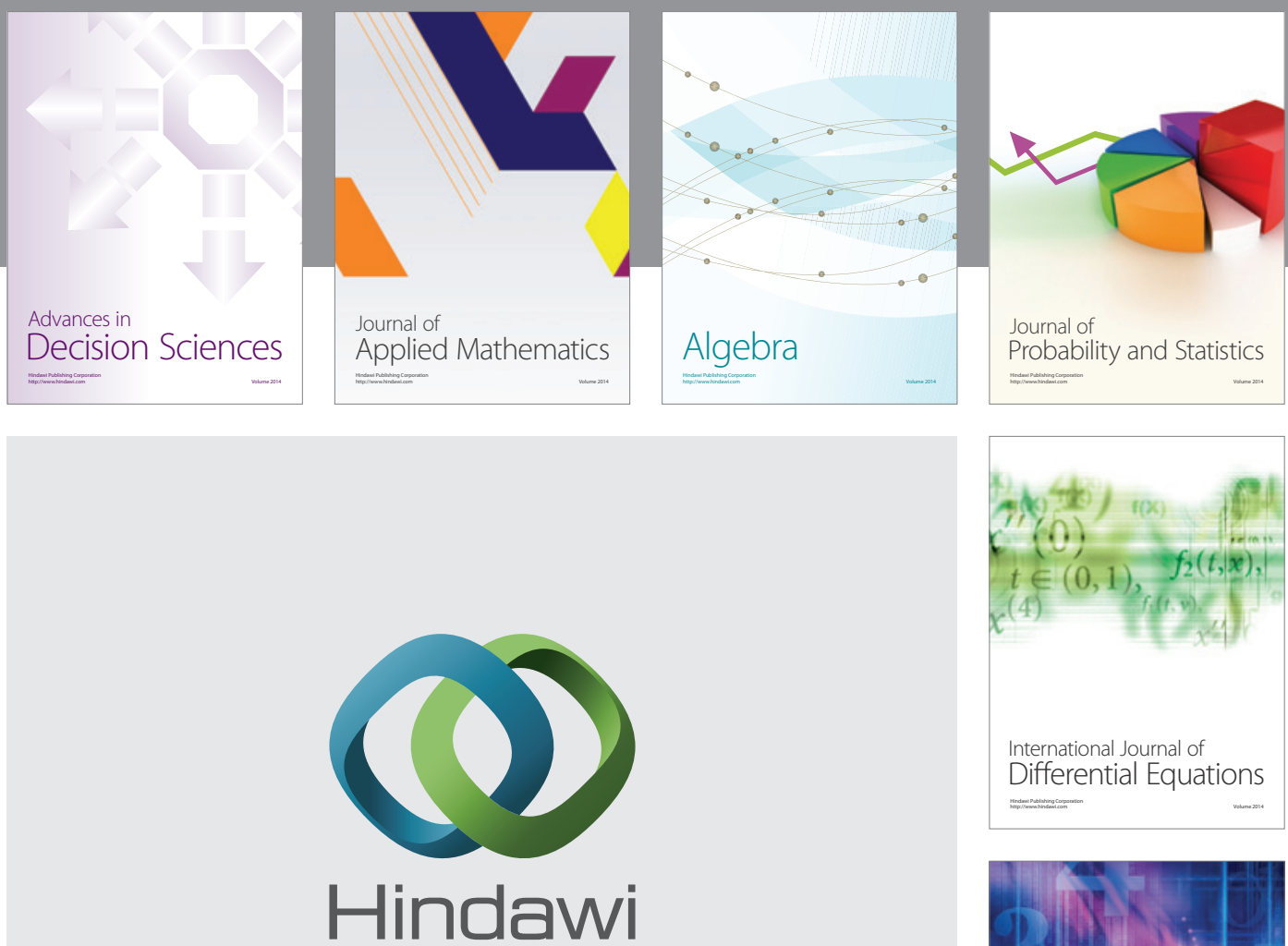

Submit your manuscripts at http://www.hindawi.com
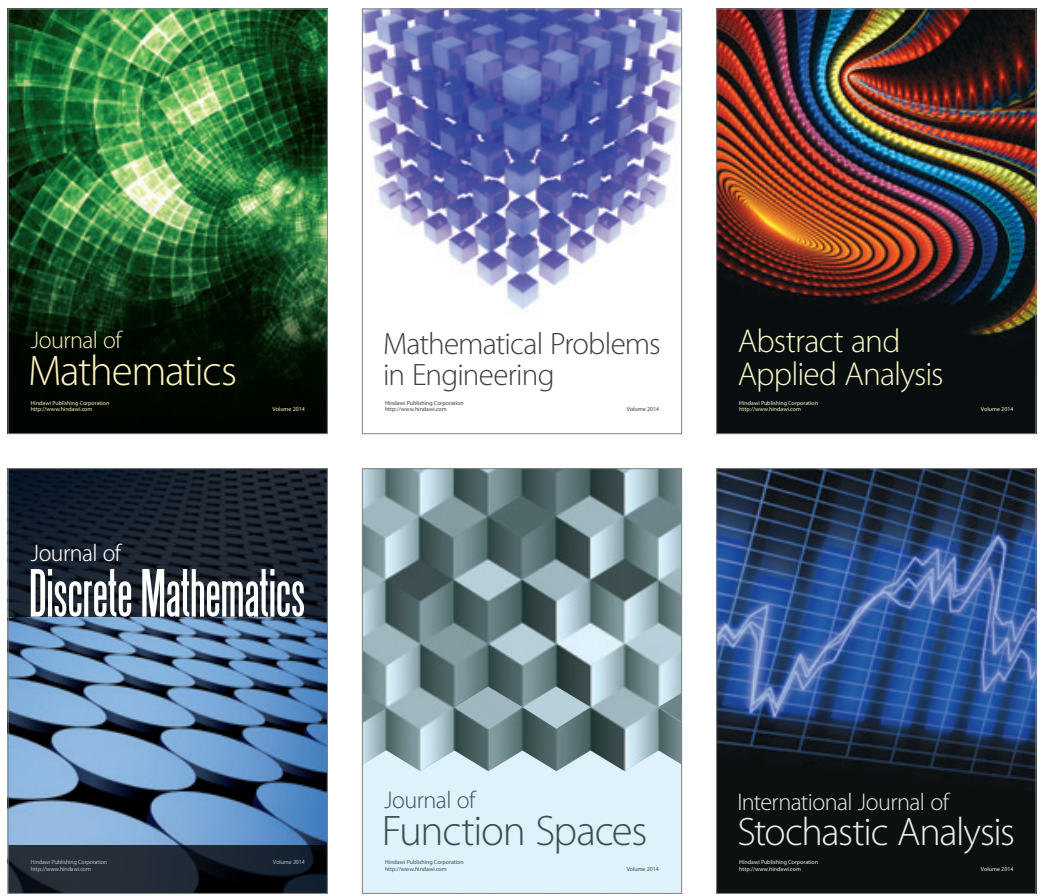

Journal of

Function Spaces

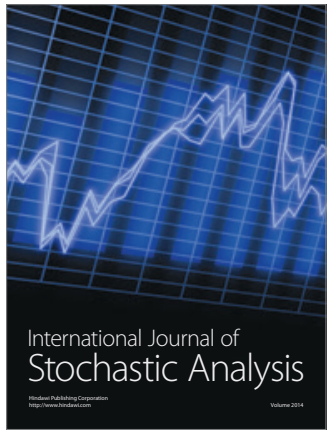

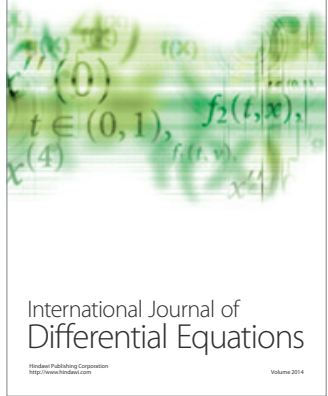
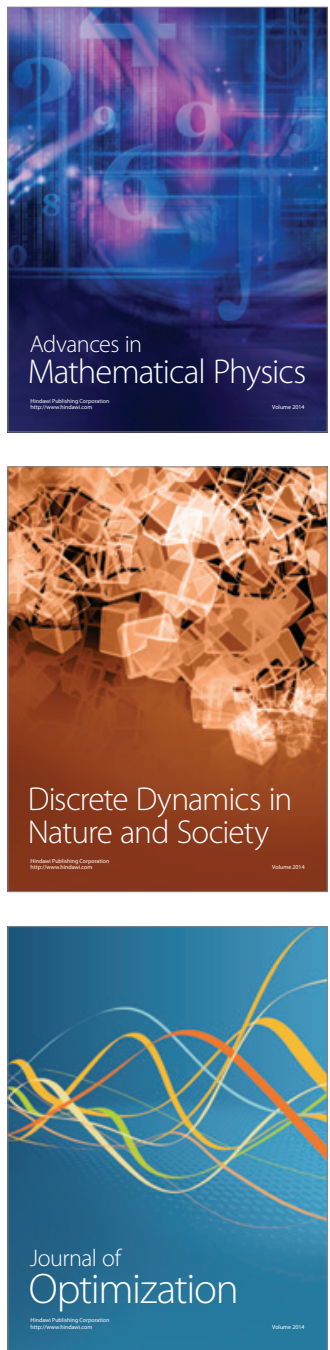